\section{Tension pneumoperitoneum: innovative decompression of this general surgical emergency}

\author{
Alun E. Jones, Dean Godfrey, \\ Guy F. Nash \\ Department of General Surgery, \\ Poole General Hospital, UK
}

\begin{abstract}
We describe the novel use of a cannula in decompressing a large tension pneumoperitoneum secondary to perforated sigmoid diverticulum, in which the patient did not subsequently require an emergency laparotomy. Needle decompression was successfully used as part of a conservative regimen, thus avoiding potentially high-risk surgery.
\end{abstract}

\section{Case Report}

A 78-year-old gentleman with severe chronic obstructive airways disease presented acutely with a five day history of severe lower abdominal pain and distension and right shoulder pain. Further questioning revealed a background of several months of change in bowel habit (alternating constipation and loose stools) but no history of weight loss. On examination he appeared sweaty and tachypnoeic, was pyrexial at $37.9^{\circ} \mathrm{C}$ and had a sinus tachycardia of 100 beats per minute. Abdominal examination revealed a tensely distended and tympanic abdomen with diffuse lower abdominal tenderness and guarding. Chest examination revealed significantly reduced air entry bibasally and scattered wheeze. Bloods revealed a normal full blood count, amylase, liver and renal function tests, but a CRP of greater than $500 \mathrm{mg} / \mathrm{L}$ and an ESR of $120 \mathrm{~mm} / \mathrm{hr}$. An erect chest X-ray revealed free gas below the diaphragm indicating a pneumoperitoneum secondary to visceral perforation (Figure 1).

He underwent an abdominal computed tomography (CT) scan which confirmed a massive pneumoperitoneum and identified the most likely source as a perforated sigmoid diverticulum (Figure 2).

Given his acute dyspnea with a tensely distended, tympanic abdomen, tension pnemoperitoneum was suspected. Clearly this gentleman was at high risk for emergency surgery. An emergency needle decompression was therefore undertaken using a 14G (brown) cannula. This immediately improved his symptoms and clinical condition as his abdomen became noticeably less distended, with a reduction in his respiratory and heart rate, and improved bilateral air entry. He continued on this trial of conservative management with needle decompression, intravenous fluids, broad spectrum antibiotics and oxygen. After $48 \mathrm{~h}$, the cannula was removed as his symptoms had largely resolved, his vital signs had normalized and his inflammatory markers were considerably improved. His clinical condition continued to improve significantly to the point of discharge five days later. No further imaging was performed.

Two months later he underwent a flexible sigmoidoscopy which confirmed sigmoid diverticular disease with no evidence of malignancy.

\section{Discussion}

Pneumoperitoneum is the pathological presence of free gas within the abdominal cavity. It commonly arises as a result of visceral perforation (e.g., gastric or duodenal ulcers, diverticular disease, malignancy, but also bladder, vagina or uterus) or iatrogenically during an endoscopic procedure (both diagnostic and interventional, e.g., polypectomy, ERCP, PEG). It is also seen as a result of abdominal trauma (both penetrating and blunt - e.g., blast injuries) or as a consequence of barotrauma in ventilated patients. Pneumoperitoneum has also been detected on CT up to eighteen days post laparotomy. ${ }^{1}$

Pneumoperitoneum may be asymptomatic and picked up incidentally, but commonly results in abdominal pain referred pain to the shoulder from diaphragmatic irritation, with evidence of localized or generalized peritonitis on abdominal examination. The diagnosis may be confirmed by the finding of free subdiaphragmmatic gas on an erect chest radiograph or free extra-luminal gas on a supine abdominal radiograph giving rise to Rigler's sign (otherwise known as the double lumen sign - the presence of free gas on both sides of the intestinal wall).

Unlike a simple pneumoperitoneum, a tension pneumoperitoneum arises when a large volume of free intra abdominal gas develops a pressure in the intraperitoneal space resulting in haemodynamic instability (by compressing the vena cava and reducing venous return) and/or respiratory failure (by elevating the diaphragm, reducing lung volumes and ventilatory capacity). Patients with a tension pneumoperitoneum have a tensely distended, tympanic abdomen, with acute dyspnea, hypotension or jugular vein congestion, and large volumes of intraperitoneal gas on imaging. In these patients the tension
Correspondence: Guy F. Nash, Department of General Surgery, Poole General Hospital, Longfleet Road, Poole, Dorset BH15 2JB, UK. Tel. +44.1202.442818 - Fax: +44.1202.442615.

E-mail: guy.nash@poole.nhs.uk

Key words: tension pneumoperitoneum.

Received for publication: 11 July 2011. Accepted for publication: 19 September 2011.

This work is licensed under a Creative Commons Attribution NonCommercial 3.0 License (CC BYNC 3.0).

(C) Copyright A.E. Jones et al., 2011

Licensee PAGEPress, Italy

Surgical Techniques Development 2011; 1:e21

doi:10.4081/std.2011.e21

requires prompt decompression, usually via emergency laparotomy, to avoid further cardiorespiratory failure or even abdominal compartment syndrome; - mesenteric ischaemia, vena caval compression and even aortic compression have been previously described. ${ }^{2}$

Since the first recorded case of tension pneumoperitoneum following resection of a

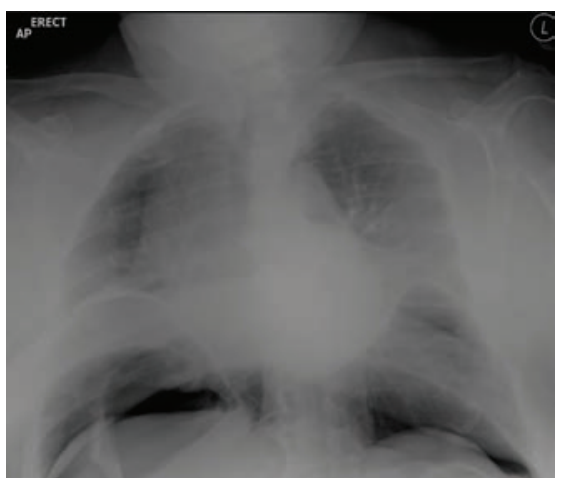

Figure 1. Erect chest radiograph showing large amount of free gas below the diaphragm.

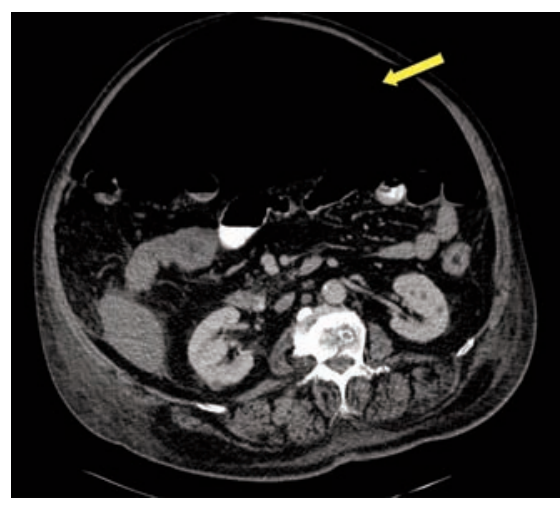

Figure 2. Abdominal computed tomography showing the massive pneumoperitoneum (arrow) 
pharyngeal diverticulum, ${ }^{3}$ there have only been a handful of case reports or small case series. ${ }^{4}$ Small perforations without tension (e.g. those arising from colonoscopic polypectomy) may be managed conservatively with intravenous fluids and broad-spectrum antibiotics. ${ }^{5}$ However, this is not always the case in one recent study, eighty percent of pneumoperitoneums arising from diagnostic colonoscopy required surgical intervention. ${ }^{6}$ In another recent study the success rate for conservative management of colonoscopyrelated pneumoperitoneum varied widely from $33 \%$ to $77 \%{ }^{7}$ In contrast, tension pneu-

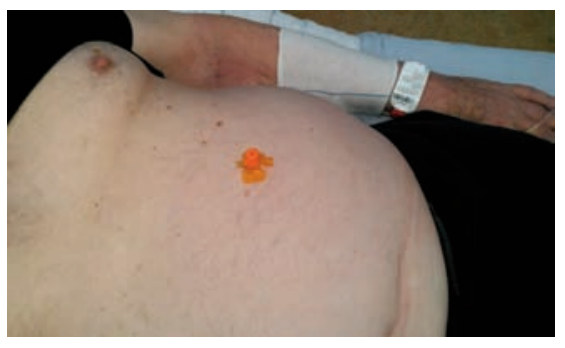

Figure 3. Percutaneous decompression of the tension pneumoperitoneum on the ward using a $14 \mathrm{G}$ cannula. moperitoneum almost invariably requires surgical intervention.

In our patient, percutaneous decompression of tension pneumoperitoneum using a 14G cannula immediately improved cardiorespiratory function (Figure 3). The cannula remained in situ for $48 \mathrm{~h}$, and in combination with broad spectrum antibiotics and fluids, this conservative management regimen allowed his condition to improve to such an extent that high-risk emergency surgery was avoided. This technique of course theoretically risks bowel injury and some would advocate at least a diagnostic laparoscopy after needle decompression.

\section{Conclusions}

We therefore suggest that percutaneous decompression of a tension pneumoperitoneum using a $14 \mathrm{G}$ cannula is a simple, cheap and effective method of immediately improving cardiorespiratory function, and can be used either as a bridge to definitive surgical treatment or as part of conservative management in patients who are poor surgical candidates.

\section{References}

1. Gayer G, Jonas T, Apter S, et al. Postoperative pneumoperitoneum as detected by CT: prevalence, duration, and relevant factors affecting its possible significance. Abdom Imaging 2000;25:301-5.

2. Olinde AJ, Carpenter D, Maher JM. Tension pneumoperitoneum. A cause of acute aortic occlusion. Arch Surg 1983;118:1347-50.

3. Conole FD, D'Angelo AA. Resection of pharyngeal diverticulum with spontaneous development of tension pneumoperitoneum. Am J Surg 1952;83:580-3.

4. Chiapponi C, Stocker U, Körner M, Ladurner R. Emergency percutaneous needle decompression for tension pneumoperitoneum. BMC Gastroenterol 2011;11:48.

5. Orsoni P, Berdah S, Verrier C, et al. Colonic perforation due to colonoscopy: a retrospective study of 48 cases. Endoscopy 1997;29: 160-4.

6. Iqbal CW, Cullinane DC, Schiller HJ, et al. Surgical management and outcomes of 165 colonoscopic perforations from a single institution. Arch Surg 2008;143:701-6.

7. Lohsiriwat V. Colonoscopic perforation: incidence, risk factors, management and outcome. World J Gastroenterol 2010;16:425-30. 